\title{
Some Numerical Curiosities about the Universe
}

\author{
Alberto Coe \\ Independent Researcher, Oviedo, Spain \\ Email: albamv8@gmail.com \\ Received 7 August 2015; accepted 22 September 2015; published 25 September 2015 \\ Copyright (C) 2015 by author and Scientific Research Publishing Inc. \\ This work is licensed under the Creative Commons Attribution International License (CC BY). \\ http://creativecommons.org/licenses/by/4.0/

c) (i) Open Access

\begin{abstract}
We try briefly the relationship between numbers and some aspects of physical reality. By means of a simple set of mathematical and physical tools what we wanted to find was dimensionless numbers that could fit with a particular symmetry. In this paper we describe a small sheaf of numerical results.
\end{abstract}

Keywords

Universe, Numerology

\section{Introduction}

In his book "Philosophy of Physics" [1] Mario Bunge states that every physical hypothesis must be expressed mathematically. But mathematical form alone won't tell us anything about the physical meaning of the formula. By itself mathematical theories are neutral with respect to any hypotheses about the actual world. Numerology can be defined as the juggling with dimensionless constants with a view to producing significant relations. Number games can occasionally lead to insights and even spark a theory proper.

Also it's known that P.A. Dirac, inspired by Eddington, Milne and others suggested a reconsideration of Cosmology based on the large dimensionless numbers that could be constructed from the fundamental constants of nature. Namely, all very large numbers occurring in nature are interconnected [2].

Besides, there is the issue of reality's stuff: Is it continuous or discrete? We assume that spacetime is granular.

\section{Method}

We'll analyze relationship among physical constants using a simple tool-kit of mathematical and physical concepts described as defined below. 
I. Symmetry $(n g+m j)$ where $n, m$ are natural numbers. And the ratio $\frac{g}{j}=\sqrt{2}$.

II. Series $S \ldots=(1+2+16+32)$ including series having the same symmetry.

III. As for the subject of the discreteness of space-time, we'll apply Avogadro's number [3]

$$
N_{\text {A }}=6.02214 \times 10^{23} \text {. }
$$

IV. An item called "sit”, associated with the topic III, representing a space-time size, arbitrarily very small.

$$
\text { Define sit }=\frac{1}{10^{n}} \text {. }
$$

(By the way you can obtain a good approximation to the number $\pi$ using properly topics I and II, as we described in a paper time ago).

\section{Some Selected Results}

Without further delay we will describe some examples of the matter at hand.

\subsection{A Geometric Beginning}

Start looking back on the topics I and II. Assign specific values to each

$$
\begin{gathered}
A=(1+\sqrt{2}) \\
B=(2+\sqrt{2}) \\
S_{\ldots=}=1+\frac{1}{2}+\frac{1}{16}+\frac{1}{32}
\end{gathered}
$$

will include two concepts more:

$$
\text { Euler's number e }=2.7182818 \ldots
$$

And Neutron-Proton mass ratio [4]

$$
\frac{N}{P}=1.001378
$$

Obtaining the following equation of evolution

$$
A B(S \ldots)^{2} \frac{N}{P} \mathrm{e}^{44} 10^{(32+16+2+1)}=\Theta_{3}
$$

Resulting volume $\Theta_{3}$ to define in paragraph 3. Now, it is easy to see that

$$
A B(S \ldots)^{2}=\Theta_{03} \text {. }
$$

This new volume $\Theta_{03}$ is defined as follow

$$
\Theta_{03}=2 \pi^{2}\left(\frac{3}{2} \sqrt{2}\right)\left(\frac{1}{\sqrt{2}}\right)^{2} .
$$

Therefore the evolution from the Torus $\Theta_{03}$ to the Torus $\Theta_{3}$

$$
\Theta_{03} \frac{N}{P} \mathrm{e}^{44} 10^{(32+16+2+1)}=\Theta_{3} .
$$

\subsection{Vacuum Expectation Value of the Higgs Field}

The Higgs field has a nonzero expectation value

$$
H E V=2.462 \times 10^{11} \mathrm{eV} \text { [5]. }
$$


The energy in electron volts of a photon after Planck equation [6] reads

$$
\begin{gathered}
E=h v \\
h=4.1356675 \times 10^{-15} \mathrm{eV} \cdot \mathrm{s} \quad[7] .
\end{gathered}
$$

Assign a specific frequency

$$
v=64 \pi \times 10^{22} \frac{1}{\mathrm{~s}} .
$$

The resulting equation relates the volume of a particular Torus $\Theta_{33}$ with the Higs vacuum value and the energy in $\mathrm{eV}$ of a photon at a specific frequency mode of vibration

$$
\Theta_{33}=\frac{H E V}{E} .
$$

Now it's interesting to see the following numerical equivalence

$$
\Theta_{33}=\Theta_{03} \sqrt{2} \times 10^{22} .
$$

Therefore rearranging the equation for the evolution described above

$$
\Theta_{33} \frac{1}{\sqrt{2}} \frac{N}{P} \mathrm{e}^{44} 10^{(29)}=\Theta_{3} .
$$

\subsection{A Light Beam Travels Three Spatial Axes for a Specified Period of Time; the Resulting Spatial Volume Is Equal to the Volume of a Particular Torus}

Volume inside a Torus

$$
V=2 \pi^{2} R r^{2}
$$

Suppose:

$$
\begin{gathered}
R=N_{A L}=6.02214 \times 10^{23} \mathrm{~m} \\
r=\frac{1}{4} R
\end{gathered}
$$

$N_{A L}$ refers to length dimension associated to Avogadro's number.

Therefore the volume of this particular Torus is defined as follow

$$
\Theta_{3}=2 \pi^{2}\left(N_{A L}\right)\left(\frac{1}{4} N_{A L}\right)^{2} .
$$

The distance light travels in one second in vacuum

$$
C_{L}=29979245 \mathrm{~m} \quad \text { [9]. }
$$

Write three spatial axes light will travel

$$
C_{L a}, C_{L b}, C_{L c} .
$$

Duration of travel in each of the axes

$$
a=10^{17} \mathrm{~s}, \quad b=10^{17} \mathrm{~s} \text { and } c=10^{12} \mathrm{~s} .
$$

(It's worth to say that according to the most recent data from the cosmic microwave background (CMB), the age of the universe is around $10^{17}$ seconds [10]).

Consequently

$$
\begin{aligned}
& C_{L a}=C_{L} 10^{17} \mathrm{~m} \\
& C_{L b}=C_{L} 10^{17} \mathrm{~m}
\end{aligned}
$$




$$
C_{L c}=C_{L} 10^{12} \mathrm{~m}
$$

Therefore polyhedron's volume

$$
C_{3}=\left(C_{L a} C_{L b} C_{L c}\right) \mathrm{m}^{3} .
$$

A simple arithmetic operation shows that

$$
\Theta_{3}=C_{3} .
$$

The results of paragraphs 1, 2 and 3 lead us to write the schematic sequence of evolution

$$
\Theta_{03} \rightarrow \Theta_{33} \rightarrow \Theta_{3} \text {. }
$$

\subsection{Relationship among $\Theta_{3}$ and Proton Compton Wavelength $\lambda_{c, p}$}

Proton Compton wavelength $\lambda_{c, p}$ is equivalent to the wavelength of a photon whose energy is the same as the rest-mass energy of the particle.

The Compton wavelength of the proton

$$
\lambda_{c, p}=1.32141 \times 10^{-15} \mathrm{~m}[11] .
$$

Relationship between $\lambda_{c, p}$ and Avogadro's number reads

$$
\lambda_{c, p}=\frac{10^{10}}{4 \pi N_{A}} .
$$

Now, consider a sphere whose radius is equal to $\lambda_{c, p}$

$$
S_{c, p}=\frac{4}{3} \pi \lambda_{c, p}^{3} .
$$

And the volumen inside a Torus defined in Equation (13)

$$
\Theta_{3}=2 \pi^{2}\left(N_{A L}\right)\left(\frac{1}{4} N_{A L}\right)^{2} .
$$

Trying to abreviate symbols

$$
\lambda_{3}=3 S_{c, p}
$$

Now will write the resulting formula

$$
\Theta_{3}=\frac{10^{30}}{(128) \lambda_{3}}
$$

(it is assumed that the volume units are canceled on both sides of the equation).

\subsection{Einstein's Gravitational Constant}

Einstein's constant denoted $K$ (kappa) is a coupling constant that appears in his field equations, whose value is given by

$$
K=\frac{8 \pi G}{C^{4}}[12]
$$

Write the volume of a particular torus

$$
\Theta_{3}=2 \pi^{2}\left(N_{A}\right)\left(\frac{1}{4} N_{A}\right)^{2}
$$

will apply the atomic mass constant

$$
m_{u}=1.66054 \times 10^{-27} \mathrm{~kg}[13] .
$$


Consider a system whose mass is

$$
m_{u v}=\frac{1}{2} m_{u} \times 10^{79}
$$

and whose acceleration is equal to

$$
a=1.00026 \times\left(\frac{1}{8}\right)^{2} \frac{\mathrm{m}}{\mathrm{s}^{2}} .
$$

Therefore the system acquires a force

$$
F_{m v}=m_{u v} a .
$$

It's easy to check that Einstein's gravitational constant units are given by

$$
\frac{\mathrm{s}^{2}}{\mathrm{~kg} \cdot \mathrm{m}} \text {. }
$$

Apply an arbitrary value to the fourth method's topic associated with the time dimension

$$
\text { sit }=\frac{1}{10^{32}} \rightarrow\left(\frac{1}{s^{2}}\right)\left(\frac{1}{10^{32}}\right)^{2} \text {. }
$$

Now, will arrange all the concepts in the following formula

$$
\Theta_{3}=F_{m v} K\left(10^{32}\right)^{2} \text {. }
$$

\subsection{Today Energy Density}

The energy density of Cosmic microwave background (CMB) in the current epoch, at a temperature $T_{0}=2.726$ $\mathrm{K}$

$$
\epsilon_{\text {rad }}\left(T_{0}\right)=4.1778 \times 10^{-14} \mathrm{~J} \cdot \mathrm{m}^{-3}[14] .
$$

Looking for a dimensionless number, write the following equation

$$
\left[\epsilon_{\text {rad }}\left(T_{0}\right)\right]\left[\Theta_{3}\right]=\frac{1}{4^{2}} h e \times 10^{92}
$$

$h$ refers to Planck constant

$$
6.62607 \times 10^{-34} \mathrm{~J} \cdot \mathrm{s} \quad[15] .
$$

The letter e refers to the Euler's number $=2.7182818 \ldots$

As for Torus volume in Equation (27) let's rewrite Equation (13)

$$
\Theta_{3}=2 \pi^{2}\left(N_{A L}\right)\left(\frac{1}{4} N_{A L}\right)^{2} .
$$

The quantity $10^{92}$ could be written differently:

$$
\left(10^{23}\right)^{4}
$$

As for the CMB, the spectral radiance peaks at

$$
v_{0}=1.602 \times 10^{11} \mathrm{~Hz} \quad[16]
$$

in the microwave range of frequencies.

We should also mention the ratio between $v_{0}$ and Euler's number

$$
\frac{e 10^{12}}{v_{0}}=12 \sqrt{2} \text {. }
$$




\subsection{Black Hole Entropy}

Starts with Bekensteing-Hawking equation [17] describing the entropy of a black hole

$$
S=\frac{1}{4} \frac{A C^{3}}{G_{N} \hbar}
$$

$C$ refers to speed of light in vacuum (already referenced in paragraph 3).

Newtonian constant of gravitation

$$
\begin{gathered}
G_{N}=6.6735 \times 10^{-11} \frac{\mathrm{m}^{3}}{\mathrm{~kg} \cdot \mathrm{s}^{2}} \quad[18] \\
\hbar=1.054572 \times 10^{-34} \mathrm{~J} \cdot \mathrm{s} \quad[19]
\end{gathered}
$$

Write the surface's area of a torus

$$
A=4 \pi^{2} R r \quad[20] .
$$

Define a certain dimension of length associated to Avogadro’s constant

$$
\text { sit }=\frac{1}{10^{35}} \mathrm{~m}
$$

which is equivalent to the Planck scale [21].

Assign to the larger radius of the torus the value

$$
R=N_{A 0} \text { sit }
$$

$N_{A 0}$ refers to an Avogadro's number “collapsed”. (This is intended to symbolize the collapse of a huge star into a black hole)

$$
N_{A 0}=6.02214 \times 10^{0} .
$$

As for the smallest radius

$$
r=\frac{1}{4} R
$$

Now write the area of this particular torus

$$
\Theta_{2}=4 \pi^{2}\left(N_{A 0} \operatorname{sit}\right)\left(\frac{1}{4} N_{A 0} \text { sit }\right) .
$$

Summarize the formula for entropy

$$
S_{\Theta_{2}}=\frac{\Theta_{2} C^{3}}{G \hbar} .
$$

Symbol $\Theta_{2}$ (Greek capital letter theta with subscript 2) stands for torus surface as was described above. We found

$$
S_{\Theta_{2}}=\frac{1}{\alpha}
$$

which in other way reads

$$
S_{\Theta_{2}} \alpha=1
$$

$\alpha$ is the fine-structure constant [22], dimensionless value associated to electromagnetic interaction, equal to $0.007297353 .$. (at zero energy).

\section{Discussion}

Since the aim of this paper is only to review some numerical approaches to the physical constants and the phys- 
ical amounts that defines the universe and reality, nobody should have to wait a theory nor one set of predictions.

We found interesting review the numerical equivalence described by Equation (13) because the accuracy of significant digits between the constants involved in both volumes. Still more when proton Compton wavelength fits numerically (20).

The above considerations are also valid for the material developed under paragraph 5 , while recognizing greater freedom (arbitrariness) in the use of the "sit".

We think appropriate to briefly comment the numerical connection among Equations (13), (20) and (26). It allow us to note a numerical linkage between one parameter belonging to Quantum mechanics, i.e. proton Compton wavelength $\left(\lambda_{c, p}\right)$ with another one parameter associated with General relativity as Einstein's gravitational constant $(k)$.

As for the items 1 and 2, considering the assumptions or theories about cosmic inflation and related matters, we have seen curious the sequence (16). Of course always from a numerical point of view.

Under the heading 6, we review two subjects. On one side is the number of photons in the Universe. Although it is still a speculative matter, estimations range around $10^{90}$, hence we have seen interesting the value obtained in the Equation (27) related to the Avogadros number i.e. $\left(10^{23}\right)^{4}$. On the other hand it should be noted that Euler's number come on the scene spontaneously while we operated with other values.

Finally, the matter of black hole entropy shows high numerical accuracy. Although we have chosen arbitrarily the type of Surface and the value of the "sit". As the values of speed of light and Planck constant are very accurate, we would have a more accurate value of G, the Newtonian constant of gravitation.

\section{Conclusion}

As for the numerology applied to Physics and Cosmology we have selected a little set of subjects. In our view they are numerically interesting. Maybe someone can see in it some kind of theoretical inspiration.

\section{Acknowledgements}

We want to thank Kelly Sang and Freya Zhang for helpful recommendations in the correct edition of this paper.

\section{References}

[1] Bunge, M. (2012) Philosophy of Physics. 36-37.

[2] Kragh, H. (2002) Quantum Generations: A History of the Physics in the Twentieth Century. 222.

[3] Olmsted, J. and Williams, G.M. (1997) Chemistry: The Molecular Science. 101.

[4] Lide, D.R. (2004) CRC Handbook of Chemistry and Physics. 1-6.

[5] (2013) Issues in Astronomy and Astrophysics. 760.

[6] Lederman, L.M. and Hill, C.T. (2004) Simmetry and the Beautiful Universe. 242.

[7] Habash, R.W.Y. (2001) Electromagnetic Fields and Radiation: Human Bioeffects and Safety. 15.

[8] Schneider, P. and Eberly, D.H. (2002) Geometric Tools for Computer Graphics. 356.

[9] Mermin, D.N. (2009) It’s about Time: Understanding Einstein's Relativity. 25.

[10] Willcox, P.J. (1992) High Energy Astronomy. 59.

[11] Woan, G. (2000) The Cambridge Handbook of Physics Formulas. Cambridge University Press, Cambridge, 8. http://dx.doi.org/10.1017/CBO9780511755828

[12] Carmeli, M. (2006) Cosmological Relativity. World Scientific, Singapore, 43. http://dx.doi.org/10.1142/6275

[13] Beddard, G. (2009) Applying Maths in the Chemical and Biomolecular Sciences: An Example-Based Approach. Oxford University Press, Oxford, 16.

[14] Liddle, A. (2013) An Introduction to Modern Cosmology. 2nd Edition, John Wiley \& Sons, Ltd., W. Sussex, 173.

[15] Clark, A.D. (2011) Physics in 5 Dimensions: Bye, Bye Big Bang. Books on Demand, 207.

[16] Anderson, R.W. (2015) The Cosmic Compendium: The Big Bang and the Early Universe. 136.

[17] Chen, T.-P. (2005) Relativity, Gravitation and Cosmology: A Basic Introduction. Oxford University Press, Oxford, 278. 
[18] Goel, V.K. (2007) Fundamentals of Physics Xi. Tata McGraw-Hill Education, New Delhi, 15-18.

[19] Bucher, J.L. (2012) The Metrology Handbook. 2nd Edition, ASQ Quality Press, Milwaukee, 147.

[20] Trishna Knowledge Systems (2010) The Pearson MAT Super Course. Pearson Education, New Delhi, 3-84.

[21] Faunce, T.A. (2014) Nanotechnology toward the Sustainocene. Pan Stanford, Singapore, 37.

[22] Scheck, F. (2014) Quantum Physics. Springer, Berlin, 6. 